\title{
Fluorescein angiographic findings in three patients with long-term intravitreal liquid silicone
}

\author{
R H GRAY, S J CRINGLE, AND I J CONSTABLE \\ From the Lions Eye Institute, 2 Verdun Street, Nedlands, Perth 6009, Western Australia
}

\begin{abstract}
SUMmaRY The long-term retinal effects of intravitreal liquid silicone (ILS) remain controversial. In this study fundus fluorescein angiographic findings in three patients with long-term ILS are presented. Sluggish or absent blood flow was observed in retinal arterioles that lay in close proximity to the ILS, and the arterioles themselves appeared narrowed. It is suggested that ILS may have a long-term effect on the retinal vasculature, owing either to direct vascular damage, secondary to damage to the neuroretina, or to the ILS preventing diffusion of oxygen into the vitreous cavity.
\end{abstract}

Despite early reports indicating that intravitreal liquid silicone (ILS) is toxic to the retina, ${ }^{1-3}$ subsequent histological and electrodiagnostic studies have not confirmed this. ${ }^{+7}$ In the absence of any clear evidence of retinal toxicity, ILS has maintained its clinical importance, particularly in monocular patients in whom other forms of retinal detachment surgery have failed.

Preliminary work in this institute has identified a possible effect of long-term ILS on the retinal vasculature of a cat, 22 months after ILS instillation. ${ }^{\mathrm{x}}$ "The silicone occupied one-third of the vitreous cavity, and an area of superior retina was found to be devoid of capillaries after an Indian ink preparation had been prepared (Fig. 1). Prompted by this observation, we performed fundus fluorescein angiography (FFA) on three patients with long-term ILS who had reasonably clear media.

\section{Materials and methods}

The case records of 55 patients undergoing retinal detachment surgery involving the use of ILS were reviewed. Three patients satisfied the following criteria: (a) silicone present in the eye for at least one year, (b) anatomically flat retina superiorly, (c) ocular media clear enough to warrant FFA, and $(d)$ patient living close enough to justify recall for angiography. Patients were examined clinically, and consent for FFA was obtained. After colour fundus

Correspondence to Dr R H Gray, Oxford Eye Hospital, Walton Street, Oxford OX2 6AW. photographs had been taken with a Zeiss or Canon fundus camera $10 \mathrm{ml}$ of fluorescein sodium was injected intravenously, and angiographic changes were recorded with a Canon $\mathrm{CF}$ 60ZA fundus camera.

\section{Case reports}

CASE 1

Male, 78 years. This patient underwent successful bilateral retinal detachment surgery in 1970. In 1975 he underwent left intracapsular cataract surgery and the retina subsequently became redetached. Proliferative vitreoretinopathy (PVR) developed, and two scleral buckling procedures were unsuccessful in reattaching the retina. In December $19804 \mathrm{ml}$ of liquid silicone ( 2000 centistokes) was injected after subretinal fluid had been drained externally and liquid vitreous drained internally. A vitrectomy was not carried out. After eight years visual acuity is hand movements in the left eye, with a clear cornea and normal intraocular tension. Silicone has not entered the anterior chamber because of anterior vitreous membranes. The upper two-thirds of the fundus is in contact with oil and the retina is flat throughout. A high $360^{\circ}$ scleral buckle is present. The right eye is pseudophakic and achieves $6 / 12$ visual acuity.

Fig. 2 shows the silicone bubble meniscus inferiorly, and superior retinal vessels appear attenuated behind the bubble. The choroid shows patchy atrophic changes. The FFA (fluorescein is white) shows minimal filling of the superior arteriole at 10 seconds 


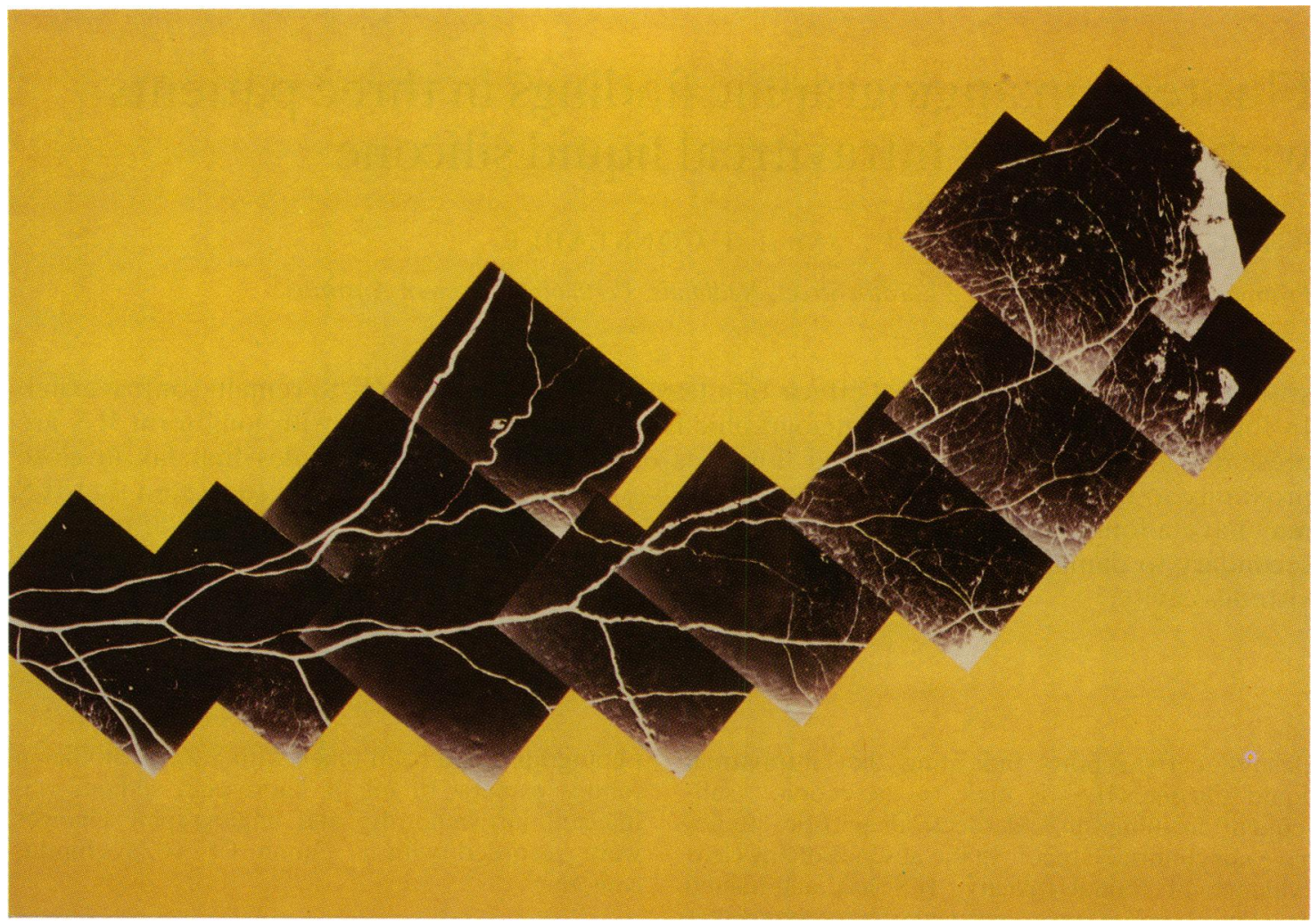

Fig. 1 Composite photograph of India ink preparation showing superior retinal vasculature of cat, 22 months after ILS instillation (optic disc to left). There is extensive small vessel non-perfusion.

(Fig. 3), and at 28 seconds the arteriole remains empty (Fig. 4). The attenuated arteriole superiorly contrasts with the larger vessels below the disc.

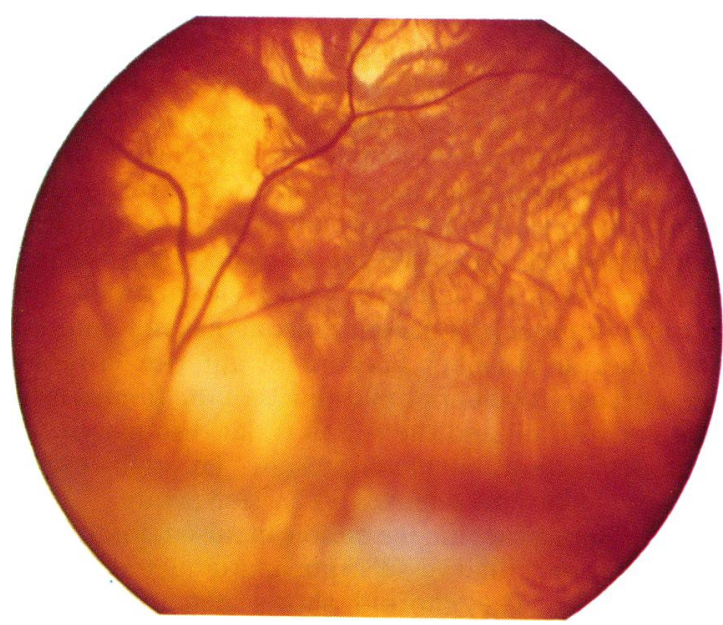

Fig. 2 Superior retina of case 1. Inferior meniscus of silicone oil visible below optic disc.
CASE 2

Female, 66 years. This patient developed a superior rhegmatogenous retinal detachment in the right eye in December 1981. Repair with a radial sponge and encircling band was initially successful, but in January 1982 the retina became redetached inferiorly with

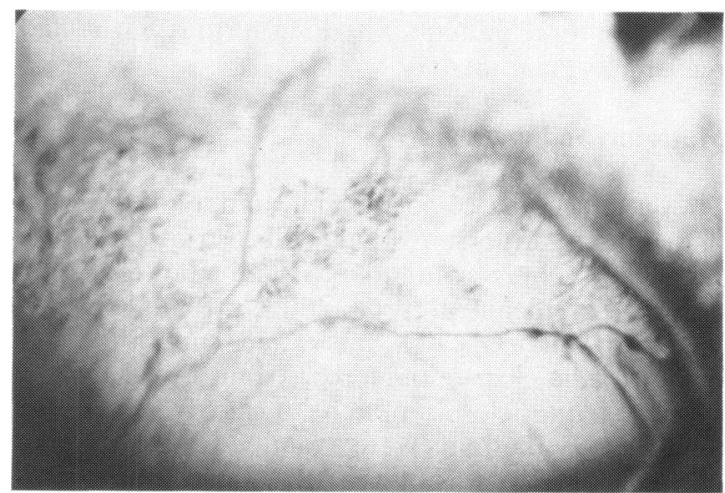

Fig. 3 FFA of case I (+10 seconds). There is minimal perfusion of the superior retinal arterioles. 


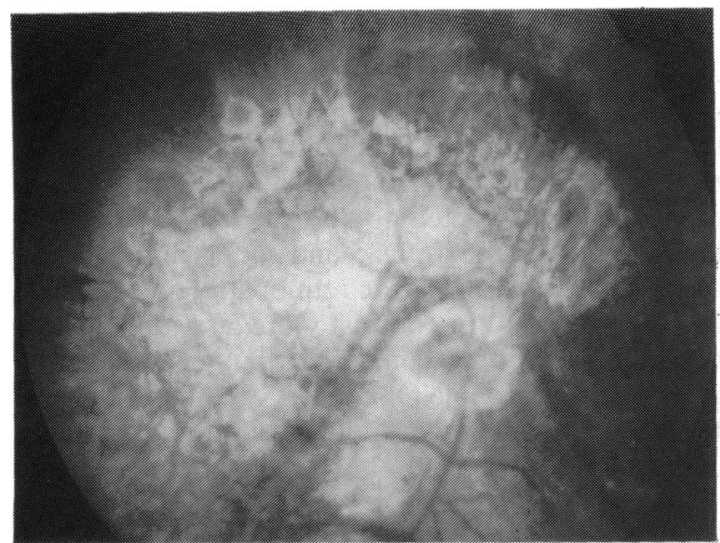

Fig. 4 FFA of case 1 (+28 seconds). Superior retinal vessel attenuation contrasts with vessels below the disc.

development of a fresh tear. After revision of the scleral buckle the retina was successfully reattached, but one month later detachment recurred with development of PVR. In February 1982, $3 \mathrm{ml}$ of liquid silicone (12500 centistokes) was injected into the vitreous cavity after external drainage of subretinal fluid and internal drainage of liquid vitreous. After six years her visual acuity is hand movements in the right eye, 6/6 left. The right cornea is clear, intraocular pressure is normal, and moderate posterior subcapsular lens opacity is present. A fibrous membrane separates the oil from the lens. The upper half of the retina is attached, the inferior half is detached and rigid. A high $360^{\circ}$ scleral buckle is present, and oil fills about one-third of the vitreous cavity.

Fig. 5 shows a deep peripheral buckle, and widespread fibrous encapsulation of the silicone bubble.

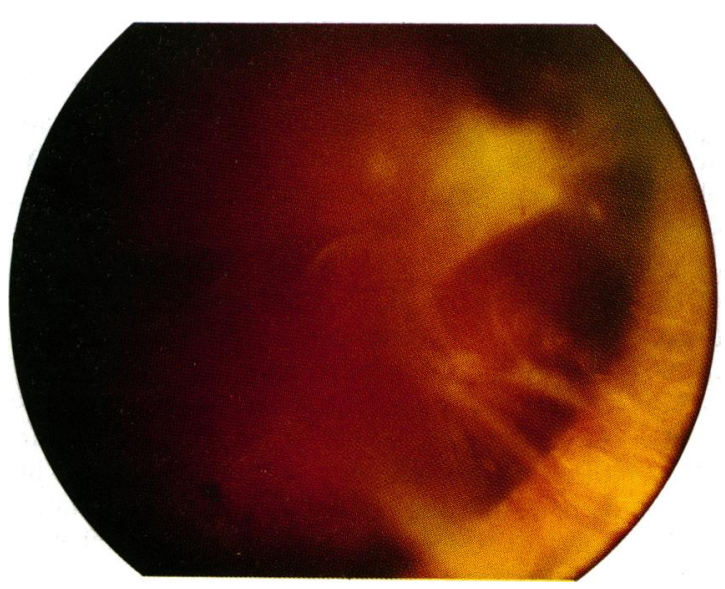

Fig. 5 Superior retina of case 2. The silicone oil is encapsulated with scar tissue. A deep peripheral indent is visible.

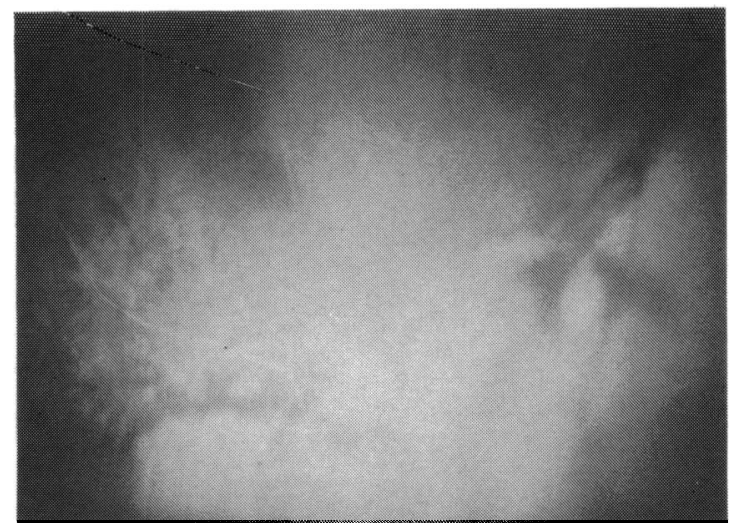

Fig. 6 FFA of case $2(+9$ seconds). A narrow retinal vessel behind the silicone oil bubble does carry the dye.

An attenuated retinal vessel behind the bubble is present. FFA shows filling of this vessel at 9 seconds (Fig. 6), and it emptied by 18 seconds.

\section{CASE 3}

Male, 33 years. This highly myopic patient developed a large inferotemporal dialysis in his right eye one month after major blunt trauma in March 1982. Conventional detachment surgery was only temporarily successful owing to the development of proliferative vitreoretinopathy, and in July 1982 a vitrectomy with gas tamponade was carried out. This failed to reattach the retina, and $3 \mathrm{ml}$ of silicone oil (2000 centistokes) was injected later that month. After six years his visual acuity is perception of light in the right eye, 6/12 in the left. The patient

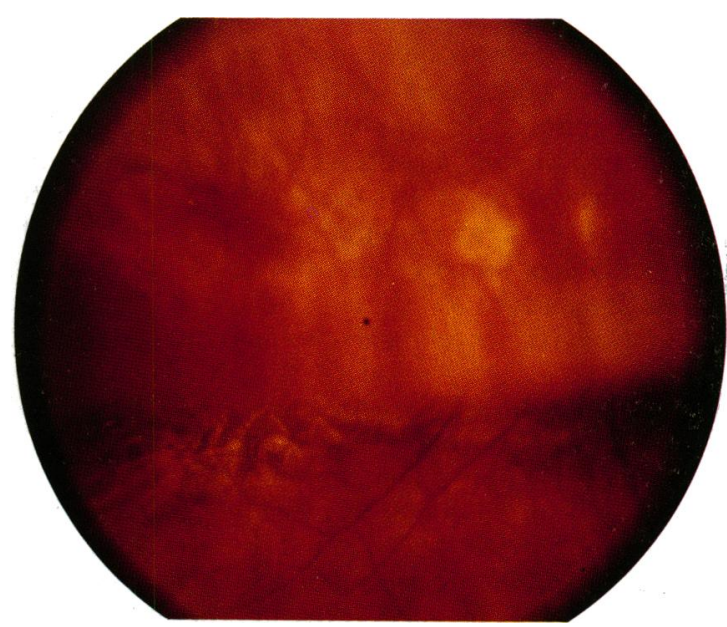

Fig. 7 Superior retina of case 3. The inferior meniscus of the silicone oil bubble obscures the disc. Superior retinal vessels appear markedly narrowed compared with those below. 


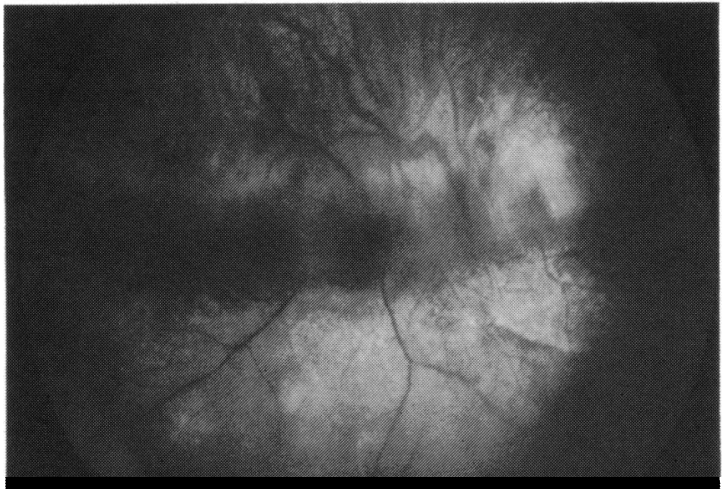

Fig. 8 FFA of case 3 (+45 seconds. There is no significant perfusion of the retinal vasculature above or below the disc.

is on topical hypotensive medications for chronic glaucoma, and a silicone emulsion is present in the anterior chamber. The iris is rubeotic. The lens is clear, and silicone fills about half the vitreous cavity. The superior two-thirds of the retina is attached, the remaining one-third shallowly detached. The optic disc is widely and deeply cupped.

Fig. 7 clearly shows the meniscus of silicone obscuring the optic disc. Retinal vessels of normal calibre below the disc contrast with the superior retinal vessels, which are hard to discern. FFA shows essentially no filling of the attenuated superior vessels. At 45 seconds there is no fluorescein visible in the retinal circulation above or below the disc (Fig. 8).

\section{Discussion}

While long-term complications of ILS such as cataract, glaucoma, and corneal decompensation are well recognised," the long-term effect of ILS on the neuroretina remains a controversial issue.

A clinical study of 407 eyes with long-term ILS stressed that useful vision remained even after 10 years in $25 \%$ of initially successful cases. "Recurrent retinal detachment was the major cause of late loss of vision. Nevertheless clinical opinion is divided on the question of early removal of ILS. McCuen et al. recommend removal between three and six months postoperatively, ${ }^{10}$ while Casswell and Gregor advocate removal in only a small number of patients. ${ }^{12}$

As a tool in the diagnosis of retinopathy electroretinography (ERG) is limited by the fact that silicone is an electrical insulator. ${ }^{67}$ Factors such as quantity of ILS injected, presence or absence of posterior vitreous detachment, and methods of measurement may explain at least part of the variable ERG findings in experimental animals. ${ }^{2.5}$
Histological studies on experimental animals have also yielded conflicting results. Lee et al.' and Mukai et al. ${ }^{23}$ demonstrated that in monkeys ILS penetrated the retina through breaks in the inner limiting membrane within hours of instillation. Retinal phospholipid was attracted to the main body of ILS, leached out of the retina, and inner retinal layer damage was noted. A toxic retinopathy was postulated on this basis. In contrast Gonvers et al. ${ }^{13}$ found that in rabbits retinal damage was limited to the outer plexiform layer, while Ober et al. ${ }^{5}$ failed to show any histological changes that could be differentiated from operated control eyes.

To our knowledge there have been no fluorescein studies on long-term human eyes with ILS except for a single case report of a patient six months after ILS instillation. ${ }^{14}$ This showed fluorescein leakage inferiorly, adjacent to intraretinal deposits of silicone that had been demonstrated on slit-lamp biomicroscopy. On the basis of this report the authors advocated removal of silicone when intraretinal droplets were clinically apparent.

Fluorescein leakage was not seen in any of our patients, but all showed a marked delay in arteriolar filling under the silicone bubble. A profound loss of retinal capillaries underlying a silicone bubble has been demonstrated in this laboratory in a single cat eye in which the ILS had been present for 22 months. ${ }^{\times}$ "While this finding remains to be confirmed, it is supported by the fluorescein changes described above. These could be due to a toxic effect of ILS on the retinal microvasculature itself, secondary to damage to the neuroretina, or possibly atrophic in response to high intraretinal partial pressures of oxygen, which would in turn be secondary to the silicone oil resisting oxygen diffusion into the vitreous cavity. ILS is known to act as a barrier to the diffusion of oxygen. ${ }^{15}$

An alternative explanation for the observed fluorescein findings is that silicone droplets had invaded the retinal arterioles and mechanically obstructed them. Mukai et al. found a single presumed silicone 'spherule' lying within a retinal arteriole during multiple sections of a monkey retina subjected to long-term ILS. ${ }^{2}$ It has been pointed out that some retinas that have been chronically detached respond by progressive narrowing of the retinal vessels when the retina is reattached, with deterioration of visual function. ${ }^{16}$ The observation of similar FFA findings above and below the ILS bubble in patient 1 , in whom the retina was fully reattached, lends weight to this argument, though the oil was mobile in this patient.

The interpretation of FFA studies on patients such as these is difficult, not only owing to the problems of visualisation, but also to the surgical procedures that 
precede the use of ILS. Despite this we believe that useful information can be gained in selected patients, particularly in regard to the question of long-term retinal toxicity involving the retinal vasculature. It is hoped that further studies will be undertaken on suitable patients, preferably in a prospective sequential trial.

We are grateful for the help of our medical photographer $\mathrm{Mr}$ Chris Barry for performing the FFA studies and preparing the photographs.

\section{References}

1 Lee P, Donovan RH, Mukai N, Schepens CL, Freeman HM Intravitreous injection of siicone: an experimental study. 1: Clinical picture and histology of the eye. Ann Ophthalmol 1969: 1: $15-25$.

2 Mukai N, Lee P, Schepens CL. Intravitreous injection of silicone: an experimental study. 2: Histochemistry and electron microscopy. Ann Ophthalmol 1972; 4: 273-87.

3 Mukai N, Lee P, Oguri M, Schepens CL. A long term evaluation of silicone retinopathy in monkeys. Can J Ophthalmol 1975; 10: 391-402.

4 Meredith TA, Lindsey DT, Edelhauser HF, Goldman AI. Electroretinographic studies following vitrectomy and intraocular silicone oil injection. Br J Ophthalmol 1985; 69: 254-60.

5 Ober RR, Blanks JC, Ogden TE, Pickford M, Minckler DS, Ryan SR. Experimental retinal tolerance to liquid silicone. Retina 1983; 3: 77-84.
6 Momirov D, Van Lith GHM, Zivojnovic R. Electroretinogram and electro-oculogram of eyes with intravitreously injected silicone oil. Ophthalmologica 1983; 186: 183-8.

7 Foerster MH, Esser J, Laqua H. Silicone oil and its influence on electrophysiologic findings. Am J Ophthalmol 1985: 99: 201-6.

8 Cringle SJ. The distribution of oxygen and ERG potentials in the isolated perfused eye. PHD thesis. University of Western Australia, 1987.

9 Constable IJ. Detachment of the retina. Aust NZ J Ophthalmol 1988; 16: 181-8.

10 McCuen BW, De Juan E, Landers MB, Machemer R. Silicone oil in vitreo-retinal surgery, part 2: results and complications. Retina 1985; 5: 198-205.

11 Chan C, Okun E. The question of ocular tolerance to intravitreal liquid silicone. A long term analysis. Ophthalmology 1986; 93: 651-60.

12 Casswell AG, Gregor ZJ. Silicone oil removal: II. Operative and postoperative complications. Br J Ophthalmol 1987; 71: 898902.

13 Gonvers M, Hornung JP, De Courten C. The effect of liquid silicone on the rabbit retina. A histologic and ultrastuctural study. Arch Ophthalmol 1986; 104: 1057-62.

14 Jalkh AE, McMeel WJ, Kozlowski IMD, Schepens CL. Silicone oil retinopathy. Arch Ophthalmol 1986; 104: 178-79.

15 De Juan E, Hardy M. Hatchell DL, Hatchell MC. The effect of intra-ocular silicone oil on anterior chamber oxygen pressure in cats. Arch Ophthalmol 1986; 104: 1063-4.

16 Constable I, Mohamed S. Tan PL. Super viscous silicone liquid in retinal surgery. Aust NZ J Ophthalmol 1982; 10: 5-11.

Accepted for publication 2 August 1989. 